\title{
Non-orthogonal Multiple-Relaxation-Time Lattice Boltzmann Simulation of Mixed Convection in Lid-Driven Porous Cavity with an Isothermally Heated Block
}

\author{
Y. Zhang, J. Bao, M. Yao, Y. Xie, Y. Huang and P. $\mathrm{Li}^{\dagger}$ \\ School of Mechanical and Electrical Engineering, Nanchang University, Nanchang 330031, China \\ †Corresponding author Address: ncudns1995z@163.com
}

(Received September 10, 2019; accepted February 22, 2020)

\begin{abstract}
Laminar mixed convection in porous cavity with an isothermally heated block had been investigated numerically by using Non-orthogonal multiple-relaxation time lattice Boltzmann method (MRT-LBM). The effects of six different arrangements of the cold sources on the characteristics of fluid flow and heat transfer had been studied. Another important influencing factor was the direction of lid-driven. We investigated the effects of four different lid-driven directions on fluid flow and heat transfer when the top and bottom walls of the cavity maintained constant cold temperature. The results show that different arrangements of the cold sources produce different numbers of vortices with the Richardson number increases. As for Top-Left, TopRight and Top-Bottom arrangements, these three arrangements always show high heat teansfer level. Additionally, the right-moving top and bottom walls exhibits best heat transfer characteristic than other three cases when $R i \leq 1$, and the case of top and bottom walls moves in the opposite directions has best heat transfer performance than other three cases when $R i>1$. When the cold sources are arranged on the upper wall of the cavity, it shows better heat transfer performance.
\end{abstract}

Keywords: Lattice Boltzmann method; Mixed convection; Lid-driven cavity; Porous media; Isothermally heated block.

$\begin{array}{ll}D & \text { Side length of the block } \\ D a & \text { Darcy number } \\ F & \text { body force } \\ f & \text { density distribution function } \\ f^{\mathrm{eq}} & \text { density equilibrium distribution function } \\ G r & \text { Grashof number } \\ g & \text { temperature distribution function } \\ g^{\text {eq }} & \text { temperature equilibrium distribution } \\ & \text { function } \\ I & \text { unit matrix } \\ K & \text { permeability } \\ k & \text { unit vector in y direction } \\ L & \text { side length of the square cavity } \\ \mathrm{M} & \text { transformation matrix } \\ \mathrm{m} & \text { velocity moments } \\ N & \text { transformation matrix }\end{array}$

$\begin{array}{ll}\frac{N u}{N u} & \text { local Nusselt number } \\ P r & \text { Prandtl number } \\ \mathrm{Q} & \text { relaxation matrix } \\ R e & \text { Reynolds number } \\ S & \text { diagonal relaxation matrix } \\ T_{h} & \text { block surface temperature } \\ T_{c} & \text { cold wall temperature } \\ u & \text { fluid velocity } \\ & \\ \varepsilon & \text { porosity of medium } \\ \rho & \text { fluid density } \\ \sigma & \text { thermal capacity ratio } \\ v & \text { kinematic viscosity } \\ \omega & \text { weight coefficients }\end{array}$




\section{INTRODUCTION}

The flow and heat transfer in lid-driven cavity with porous media has many applications in science and engineering, such as solid-matrix heat exchangers, food processing, separation process, nuclear reactors, crystal growth et al. (Iwatsu et al.1993; Vafai, 2006). In recent years, many scholars have studied mixed convection problem driven by the combination of buoyancy and shear forces in porous cavity, and obtained important results. Sivakumar et al. (2010) studied mixed convection in a lid-driven cavity with different lengths at heating portions and different locations. They found that when the heat portion was located in the middle or top of the hot wall of the cavity, the heat transfer rate was enhanced on reducing heat portion. Oztop et al. (2009) investigated the heat transfer characteristics of mixed convection for a lid-driven cavity with a circular body. They studied the influence of the Richardson number, the diameter of the inner cylinder, and the center and position of the inner cylinder on fluid flow and heat transfer. They concluded that the most effective parameter for flow field and temperature distribution was the direction of the moving lid. Cheng et al. (2010) investigated the influence of temperature gradient orientation on fluid flow and heat transfer in a lid-driven cavity. It has been found by research that the fluid flow, heat transport processes, and heat transfer rates were affected by both Richardson number and direction of temperature gradient in the cavity.

The Lattice Boltzmann (LB) method, a mesoscopic numerical simulation method, has developed in recent years. Compared with traditional numerical method, it has the advantages of clear physical image, easy boundary condition processing and good parallel performance. It has achieved great success in the simulation of fluid flows and model physics in fluids (Khali et al. 2013; Li et al. 2015; Liu and He, 2015; Nebbali et al. 201). Due to its dynamic nature and unique computational capability, the LB method has also been applied to discuss the characteristics of fluid flow and heat transfer in porous media. Zhao et al. (2010) used LB method to study natural convection in porous media. They found that overall heat transfer was enhanced with the porosity and cell size decreasing. Li et al. (2018) simulated natural convection phenomenon in porous cavity through LBM method. All these previous studies haven shown that the effectiveness of using LB method to simulate flow and heat transfer in porous cavity. In recent years, some researchers studied mixed convection in porous cavity through LB method.
Chai et al. (2007) used LB method to study the problems of mixed convection in a lid-driven porous cavity. Arun et al. (2015) used LB method to study the characteristics of a two sided lid driven cavity. They studied the effect of various Reynolds numbers (100, 1000, 2000 and 5000) and aspect ratios (1,2 and 4) on flow characteristics, it was observed that secondary and tertiary vortices appeared in the cavity when Reynolds number was higher. However, at lower Reynolds number, only primary vortices were observed in the cavity.

Although many scholars have studied laminar mixed convection in empty cavity under various temperature boundary conditions and different configurations, few studies have been devoted for the problem of mixed convection in a cavity with internal heated block. This problem has important applications in broad fields such as electronic cooling system, food processing, float glass production, oil extraction. Islam et al. (2012) studied mixed convection in lid-driven cavity within an isothermally heated square block. They found that the main Nusselt number does not change significantly with Richardson number $(R i)$ increasing when the value of $R i<1$ for any size of isothermal heated block placed anywhere in the cavity. The main Nusselt number increased obviously with Richardson number when its value exceeds 1. Billah et al. (2011) analyzed mixed convection in a lid-driven cavity with a centrally heated circular hollow cylinder. Kalteh et al. (2014) studied nanofluid mixed convection in a lid-driven cavity with heated triangular block. Gangawane et al. (2017) investigated heat transfer characteristics of a cavity in which centered triangular block was maintained a constant wall temperature or constant heat flux condition. They also established functional relationship between the average Nusselt number and the flow governing dimensionless parameters.

Above all, for porous cavity with internal heated block, the effects of different arrangements of cold sources and different lid-driven models on fluid flow and heat transfer have not been carefully researched in previous literatures. In this paper, non-orthogonal Multiple-Relaxation-Time Lattice Boltzmann Method is used to study laminar mixed convection in a lid-driven porous square cavity containing isothermally heated square block under different arrangements of cold sources and different lid-driven directions. Firstly, under fixed top liddriven condition, we investigate the effects of six different arrangements of the cold sources on fluid flow and heat transfer with a wide range of Richardson number. Then under fixed thermal boundary conditions, four different lid-driven directions are investigated. 

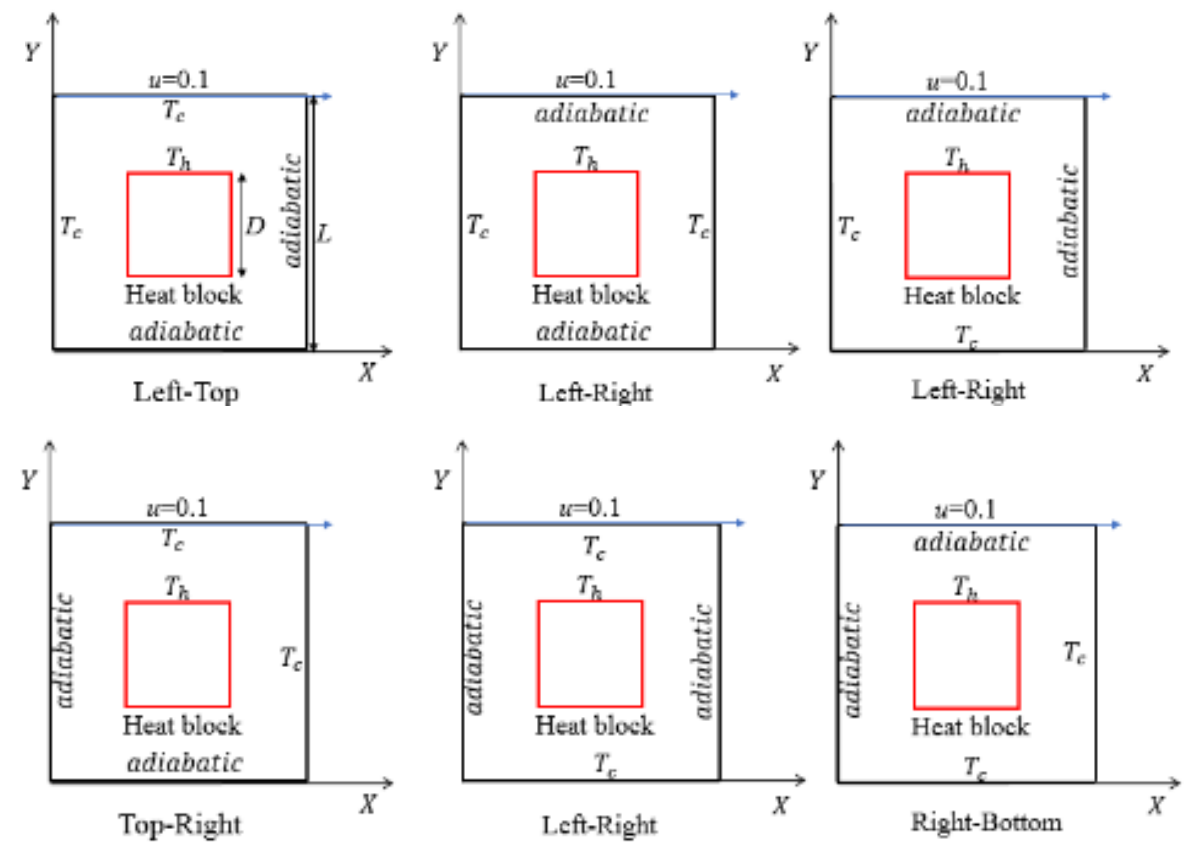

Fig. 1. Physical model for six different arrangements of cold sources.

\section{Problem Description AND NUMERICAL METHOD}

The details of the cavity physical model within isothermally heated square block are shown in Fig.1. The length of the side of the square cavity is $L$ while the block size is $D$. The block is located in the center of the square cavity and accounts for 30 percent of the square cavity size. When studying different arrangements of the cold sources, only top wall moves rightwards and other walls remain stationary for all studied cold conditions. The case of Top-Left means that the top and left walls are keeping low temperature boundary condition while bottom and right walls are thermally insulated. The same is true for other five cold source configurations. Block surfaces maintain high temperature condition, and all four surfaces are stationary. The fluid flow of the cavity is assumed to be two- dimensional, impressible Newtonian fluid. The viscous dissipation effects and radiation heat transfer are neglected. The heat transfer in the block is ignored.

The macroscopic governing equation for fluid flow and heat transfer in porous media can be written as a dimensionless form (Chai et al. 2007):

$\nabla u=0$

$\frac{\partial u}{\partial t}+(u \cdot \nabla)\left(\frac{u}{\varepsilon}\right)=-\nabla(\mathrm{P} \varepsilon)+\frac{1}{R e_{e}} \nabla^{2} u+F$ $\sigma \frac{\partial T}{\partial t}+u \cdot \nabla T=\frac{1}{\operatorname{PrRe}} \nabla^{2} T$

where $u, P$ and $T$ are the volume averaged fluid velocity, pressure and temperature, respectively; $\varepsilon$

is the porosity of medium, $P r$ is the Prandtl number, $R e_{\mathrm{e}}$ is the effective Reynolds number; $\sigma$ is the thermal capacity ratio between the inter-porosity fluid and the porous medium solid skeleton; $\boldsymbol{F}$ is the total body force, it can be written in dimensionless form

$\boldsymbol{F}=-\frac{\varepsilon}{D a R e} u-\frac{\varepsilon F_{\varepsilon}}{\sqrt{D a}}|u| u+\frac{\varepsilon G r}{\operatorname{Re}_{e}^{2}} k T$

where $D a$ is the Darcy number, Re is the Reynolds number which we assume that $R e$ is equal to $R e_{e}, k$ is the unit vector in y direction. $G r$ is the Grashof number, it can be defined as:

$G r=R i * R e^{2}$

The geometric function $F_{\varepsilon}$ and permeability $K$ can be expressed as (Vafai et al. 2006):

$F_{\varepsilon}=\frac{1.75}{\sqrt{150 \varepsilon^{3}}}, K=\frac{\varepsilon^{3} d_{p}^{2}}{150(1-\varepsilon)^{2}}$

where $d_{p}$ is the diameter of the spherical particle.

Several dimensionless parameters involved in fluid flow and heat transfer in porous medium are given by: 


$$
R i=\frac{g \beta\left(T_{h}-T_{c}\right) L}{U_{0}^{2}}, R e=\frac{U_{0} L}{v}, \operatorname{Pr}=\frac{v}{\alpha}, D a=\frac{K}{L^{2}}
$$

The average Nusselt number $\overline{N u}$ at hot wall can be defined as:

$$
\overline{N u}=\frac{1}{\Delta T} \int_{0}^{L} N u(y) d y
$$

where $N u$ is the local Nusselt number at hot wall, $N u(y)=-L(\partial T / \partial x)_{\text {wall }} / \Delta T$.

\section{MRT-LB MODEL FOR THERMAL FLOWS IN POROUS MEDIA}

\subsection{Flow Field}

The lattice Boltzmann equation describes the spatiotemporal evolution of the gas molecule distribution function, and the macroscopic flow parameters are obtained by the relationship between the macroscopic flow variables and the distribution function (Jonas, 2008; Koosukuntla, 2011). The evolution equation of the velocity distribution of the non-orthogonal transformation matrix MRT-LB model is (Li et al. 2010):

$$
\begin{aligned}
& f_{i}\left(x+e \delta_{t}, t+\delta_{t}\right)-f_{i}(x, t)=-\boldsymbol{M}^{-1} \cdot\left\{\boldsymbol{S} \cdot\left[m-m^{e q}\right]_{x, t}-\right. \\
& \left.\delta_{t}\left(I-\frac{\boldsymbol{S}}{2}\right) \cdot F_{x, t}\right\}_{t}
\end{aligned}
$$

where $f$ is the density distribution function, $\boldsymbol{M}$ is the non-orthogonal transformation matrix in velocity space, $S$ is the diagonal relaxation matrix, $I$ is the unit matrix, $\boldsymbol{F}$ is the force item; $m$ and $m^{e q}$ are the velocity moments and the corresponding equilibrium moments, respectively, they can written as

$$
\boldsymbol{m}=\boldsymbol{M} \cdot f, \boldsymbol{m}^{e q}=\boldsymbol{M} \cdot f^{e q}
$$

In the model of D2Q9 as shown in Fig.2, the discrete velocities of the D2Q9 lattice are defined as(Qian et.al,1992):

$$
e_{i}=\left\{\begin{array}{ll}
(0,0), & i=0 \\
(1,0) c,(0,1) c,(-1,0) c,(0,-1) c, & i=1-4 \\
(1,1) c,(-1,1) c,(-1,-1) c,(1,-1) c, & i=5-8
\end{array}\right\}
$$

where $c=\delta_{x} / \delta_{t}$ is the lattice speed, $\delta_{x}$ and $\delta_{t}$ are the discrete time step and lattice spacing, respectively. In this work, the lattice speed is sent to be 1. For non-orthogonal transformation matrix $\boldsymbol{M}$,it can be defined as (Premnath et al. 2009):
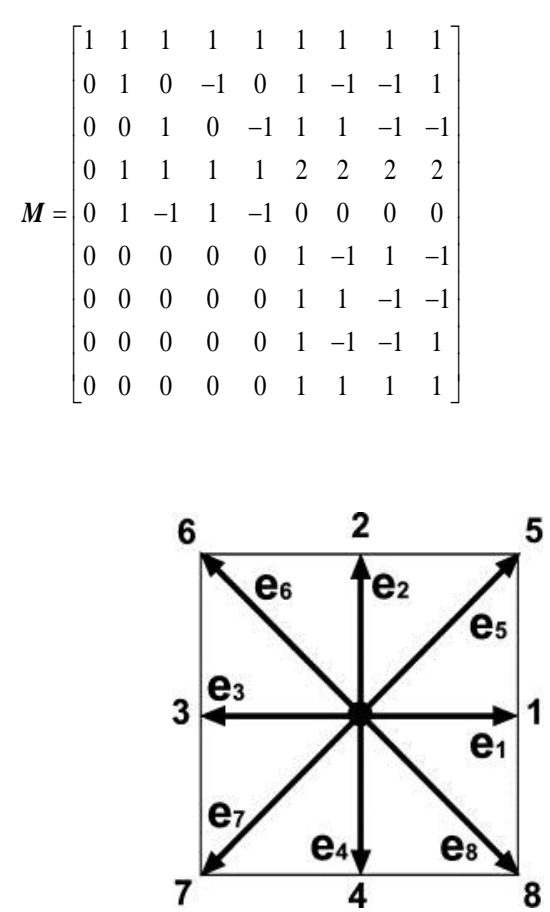

Fig. 2. A D2Q9 lattice.

Through the matrix $\boldsymbol{M}$, the moment vector is given by:

$\boldsymbol{m}=\left(\rho, e, \varepsilon, j_{x}-\frac{\delta_{t}}{2} \rho F_{x}, q_{x}, j_{y}-\frac{\delta_{t}}{2} \rho F_{y}, q_{y}, p_{x x}, p_{x y}\right)^{T}$

where $j_{x}$ and $j_{y}$ are $\mathrm{x}$-and $\mathrm{y}$ - components of the momentum, $j=\left(j_{x}, j_{y}\right)=\rho u$, respectively, $\quad F_{x}$ and $F_{y}$ are $x$ - and $y$-components of the total body force $\boldsymbol{F}$, respectively.

The equilibrium moments $m^{e q}$ for the moments is defined as:

$$
\begin{aligned}
& m_{0}^{e q}=\rho, m_{1}^{e q}=\rho u_{x}, m_{2}^{e q}=\rho u_{y}, m_{3}^{e q}=\frac{2 \rho}{3}+\frac{\rho\left(u_{x}^{2}+u_{y}^{2}\right)}{\varepsilon}, \\
& m_{4}^{e q}=\frac{\rho\left(u_{x}^{2}-u_{y}^{2}\right)}{\varepsilon}, m_{5}^{e q}=\frac{\rho u_{x} u_{y}}{\varepsilon}, \\
& m_{6}^{e q}=\frac{u_{y}}{3}, m_{7}^{e q}=\frac{u_{x}}{3}, m_{8}^{e q}=\frac{\rho}{9}+\frac{\rho\left(u_{x}^{2}+u_{y}^{2}\right)}{3 \varepsilon}
\end{aligned}
$$

The evolution of the MRT-LB equation consists of two steps, the collision step and steaming step. The first is the moment space collision:

$$
m^{*}(x, t)=m(x, t)-\left.S\left(m-m^{e q}\right)\right|_{(x, t)}+\delta_{t}\left(I-\frac{S}{2}\right) F
$$

where $m^{*}$ is the moment space distribution function after the collision.

The components of the forcing term $\boldsymbol{F}$ in the moment space are given by: 


$$
\begin{aligned}
& F_{0}=0, \quad F_{1}=\rho F_{x}, F_{2}=\rho F_{y}, F_{3}=\frac{2 \rho\left(u_{x} F_{x}+u_{y} F_{y}\right)}{\varepsilon}, \\
& F_{4}=\frac{2 \rho\left(u_{x} F_{x}-u_{y} F_{y}\right)}{\varepsilon}, F_{5}=\frac{2 \rho\left(u_{x} F_{y}+u_{y} F_{x}\right)}{\varepsilon}, \\
& F_{6}=\frac{1}{3} \rho F_{y}, F_{7}=\frac{1}{3} \rho F_{x}, F_{8}=\frac{2}{3} \frac{\rho\left(u_{x} F_{y}+u_{y} F_{x}\right)}{\varepsilon}
\end{aligned}
$$

The streaming step is completed in velocity space:

$$
f_{i}\left(x+e_{i}, t+\delta_{t}\right)=f_{i}^{*}(x, t)
$$

where $f^{*}=M^{-1} m^{*}$

The equilibrium distribution function of the velocity field is defined as (Guo et al. 2002):

$$
f_{i}^{e q}=\omega_{i} \rho\left[1+\frac{e_{i} \cdot u}{c_{s}^{2}}+\frac{u u:\left(e_{i} e_{i}-c_{s}^{2} I\right)}{2 \varepsilon c_{s}^{4}}\right]
$$

where $c_{s}=1 / \sqrt{3}$ is the lattice sound speed of D2Q9 model and $\omega_{i}$ are weight coefficients, they are given by:

$$
\begin{aligned}
& \omega_{0}=4 / 9, \omega_{1}=1 / 9, \omega_{2}=1 / 9, \omega_{3}=1 / 9, \omega_{4}=1 / 9, \\
& \omega_{5}=1 / 36, \omega_{6}=1 / 36, \omega_{7}=1 / 36, \omega_{8}=1 / 36,
\end{aligned}
$$

The macroscopic density and velocity of the fluid are given by:

$$
\begin{aligned}
& \rho=\sum_{i=0}^{8} f_{i}=\sum_{i=0}^{8} \bar{f}_{i} \\
& \rho u=\sum_{i=0}^{8} e_{i} f_{i}=\sum_{i=0}^{8} e_{i} \bar{f}_{i}+\frac{\delta_{t}}{2} \rho \boldsymbol{F}
\end{aligned}
$$

By introducing a temporary velocity, the macroscopic velocity of the fluid can be obtained:

$$
u=\frac{v}{c_{0}+\sqrt{c_{0}^{2}}+c_{1}|v|}
$$

where

$v=\sum_{i=0}^{8} e_{i} f_{i} / \rho_{0}+\frac{\delta_{t}}{2} \varepsilon G, c_{0}=\frac{1}{2}\left(1+\varepsilon \frac{\delta_{t}}{2} \frac{v}{K}\right), c_{1}=\varepsilon \frac{\delta_{t}}{2} \frac{F_{\varepsilon}}{\sqrt{K}}$

\subsection{Temperature Field}

In the solution of temperature field, the nonorthogonal MRT-LB equation of the twodimensional temperature field is given by:

$$
g_{i}\left(x+e_{i} \delta_{t}\right)-g_{i}(x, t)=-\left.\left(\boldsymbol{N}^{-1} \boldsymbol{Q N}\right)_{i j}\left(g_{j}-g_{j}^{e q}\right)\right|_{(x, t)}
$$

where $g$ and $g^{e q}$ are the distribution functions of temperature and the temperature equilibrium, respectively; $\quad \boldsymbol{N}$ is the non-orthogonal transformation matrix and $N^{-1}$ is its inverse matrix, $Q$ is the relaxation matrix.

In the D2Q5 model, the direction vector of discrete velocity is given by:

$e_{i}= \begin{cases}(0,0), & i=0 \\ (\cos [(i-1) \pi / 2], \sin [(i-1) \pi / 2] c, & i=1-4\end{cases}$

Matrix $N$ can be defined as (Liu et al. 2016):

$\boldsymbol{N}=\left[\begin{array}{ccccc}1 & 1 & 1 & 1 & 1 \\ 0 & 1 & 0 & -1 & 0 \\ 0 & 0 & 1 & 0 & -1 \\ 0 & 1 & 1 & 1 & 1 \\ 0 & 1 & -1 & 1 & -1\end{array}\right]$

Through the transformation matrix $N$, the collision step of the MRT-LB equation can be carried out in the moment space:

$$
n^{*}(x, t)=n(x, t)-\left.\boldsymbol{Q}\left(n-n^{e q}\right)\right|_{(x, t)}
$$

where $n^{*}$ is the moment of collision, and $n=N g$, $n_{0}$ is the only conserved quantity and the temperature can be computed by:

$\sigma T=n_{0}=\sum_{i=0}^{4} g_{i}$

The equilibrium moments $n^{e q}\left(n^{e q}=N g^{e q}\right)$ are defined as:

$n_{0}^{e q}=T, n_{1}^{e q}=u_{x} T, n_{2}^{e q}=u_{y} T$,
$n_{3}^{e q}=\varpi \sigma T, n_{4}^{e q}=0$

where $\varpi$ is a constant. The diagonal relaxation matrix $\boldsymbol{Q}$ is given by:

$\boldsymbol{Q}=\operatorname{diag}\left(\zeta_{0}, \zeta_{\alpha}, \zeta_{\alpha}, \zeta_{3}, \zeta_{4}\right)$

After the collision step, the streaming step can be executed in velocity space:

$g_{i}\left(x+e_{i} \delta_{t}, t+\delta_{t}\right)=g_{i}^{*}(x, t)$

where $g^{*}=N^{-1} n^{*}$.The equilibrium distribution $g^{e q}$ in velocity space is given by:

$g_{i}^{e q}=\left\{\begin{aligned}(1-\omega) \sigma T, & i & =0 \\ \frac{1}{4} \omega \sigma T+\frac{1}{2}\left(e_{i} \mathrm{u}\right) T, & i & =1-4\end{aligned}\right.$

\section{Grid Independency Study And CODE VALIDATION}

In order to find optimal grid size to simulate mixed convection in lid-driven porous cavity with isothermally heated block, numerical computations have been carried out with four cases of uniform grid: $80 \times 80 、 120 \times 120 、 160 \times 160 、 200 \times 200$. In the flow field, we use bounce-back method to 
Table 1 Grid independence study for $G r=10^{5}, R i=10, \varepsilon=0.4$

\begin{tabular}{|cc|cc|}
\hline $\mathrm{n}$ & Grid size & $\overline{N u}$ & $\Delta$ \\
\hline 1 & $80 \times 80$ & 5.58 & - \\
\hline 2 & $120 \times 120$ & 5.66 & $1.434 \%$ \\
\hline 3 & $160 \times 160$ & 5.70 & $0.707 \%$ \\
\hline 4 & $200 \times 200$ & 5.73 & $0.526 \%$ \\
\hline
\end{tabular}

Table 2 Comparison of average Nusselt number at hot wall with previous studies

\begin{tabular}{|c|rr|c|}
\hline $\mathrm{Re}$ & Present & Iwatsu et al.(1993) & Al-Amiri et al.(2000) \\
\hline 100 & 2.03 & 1.94 & 2.01 \\
\hline 400 & 3.87 & 3.84 & 3.91 \\
\hline 1000 & 6.20 & 6.33 & 6.33 \\
\hline
\end{tabular}

Table 3 Comparisons of maximum and minimum values of the horizontal and vertical velocities in the center lines of the square cavity with previous studies

\begin{tabular}{|c|c|c|c|c|c|c|}
\hline & \multicolumn{3}{|c|}{$R i=1.0 \times 10^{-2}$} & \multicolumn{3}{c|}{$R i=6.25 \times 10^{-4}$} \\
\hline & Present & $\begin{array}{c}\text { Iwatsu et al. } \\
(1993)\end{array}$ & $\begin{array}{c}\text { Al-Amiri et al. } \\
(2000)\end{array}$ & Present & $\begin{array}{c}\text { Iwatsu et al. } \\
(1993)\end{array}$ & $\begin{array}{c}\text { Al-Amiri et al. } \\
(2000)\end{array}$ \\
\hline$u_{\max }$ & 1.0000 & 1.0000 & 1.0000 & 1.0000 & 1.0000 & 1.0000 \\
\hline$u_{\min }$ & -0.2040 & -0.2037 & -0.2122 & -0.3077 & -0.3197 & -0.3099 \\
\hline$u_{\max }$ & 0.1699 & 0.1699 & 0.1765 & 0.2825 & 0.2955 & 0.2866 \\
\hline$u_{\min }$ & -0.2423 & -0.2448 & -0.2506 & -0.4397 & -0.4459 & -0.4363 \\
\hline
\end{tabular}

achieve the no-slip boundary condition and use boundary condition with known velocity to achieve slip boundary condition (Zou and He, 1997). In the temperature field, we use constant temperature boundary condition. The results of average Nusselt number on the hot walls of the isothermally heated block under different grid size are presented in Table 1. As seen in Table1, when the grid size is $120 \times 120$, the average Nusselt number on hot wall changed by less than $0.8 \%$ with the grid size increasing. In order to decrease the calculating time, the grid size of $120 \times 120$ is selected for simulating the follow analysis.

In this paper, numerical simulations of mixed convection in square cavity with a driving lid are carried out to validate the correctness of the nonorthogonal MRT method. The results are compared with the numerical results reported in previous studies in the absence of porous media and heat generation for $P r=0.71$. The top wall of the cavity keeps hot constant temperature and the bottom wall keeps cold constant temperature. The left and right wall maintain adiabatic condition. The comparison results of the average Nusselt number at hot wall are listed in Table 2. The comparison results of the $\mathrm{u}$ - velocity in the vertical mid-plane and the vvelocity in the horizontal mid-plane are shown in the Table 3.

\section{Results ANd Discussion}

In this work, a numerical analysis on mixed convection in lid-driven square cavity with isothermally heated block is carried out. A main consideration is given to the impact of the cold source placement, the Richardson number and different lid-driven patterns. In order to find the optimal cold source arrangement, only the top wall have a rightward velocity $u$. All numerical computations are performed at a fixed Grashof number of $10^{5}$ for air with Prandtl number of 0.71 and the porosity of medium is 0.4 .

\subsection{Different Cold Source Placement under a Lid-Driven}

Different forms of cold source arrangement 
greatly affect the flow of fluid and heat transfer in the cavity. In order to find the best cooling effect, in this section, we study the heat transfer strength of six different arrangements of the cold sources. The considered values of the Richardson number are $0.1,1.0$ and 10 , which correspond to the regimes of forced convection, mixed convection, natural convection dominated flow. Figures 3, 5, 7 show the isotherm and streamline diagrams for six arrangements of cold sources with $R i=0.1$, $1.0,10$, respectively. The variation of the local Nusselt number along the hot wall of the ted block are shown in Figs. 4, 6, 8 with $R i=0.1,1.0$, 10 , respectively. It starts from upper right corner of the isothermally heated block, and follow the right, bottom, left of the block, and then terminate at the top of the block. When $R i=0.1$, forced convection dominates fluid flow and heat transfer in the cavity, the flow diagrams are roughly same under six different arrangements of cold sources as a result of strong convection, it behaves as a flow pattern of single vortex. All vortex centers act on the upper right corner of the block. For all arrangements, small vortex can also be observed in the lower left and lower right corners of the cavity from Fig.3, which is the characteristic of the lid-driven cavity. Due to the presence of the lid-driven in the top wall, the isotherms bend to the upper right corner of the cavity. It can be seen in Fig.3, the isotherms are more clustered towards the top moving lid and the block surface, which indicating the development of thin boundary layers with large temperature gradients along these surfaces. Due to the arrangement of the cold sources in the top and right walls, it allows the hot fluid clustered in the upper right corner of the cavity to quickly dissipate heat. Therefore, the arrangement of Top-Right shows best heat transfer characteristic. Through comparison, it is found that the arrangements of Top-Right, TopBottom, Top-Left have better heat transfer effects than other three arangements in the cavity. And the arrangement of Left-Bottom shows the worst heat transfer effect. To investigate the effects of the cold source location on heat transfer in the cavity, Fig.4 shows the values of local Nussult number with $R i=0.1$. The Top-Right arrangement shows the largest local Nusselt number on the both right and bottom surfaces of the block, and the Left-Bottom arrangement has a minimum local Nusselt value on all four surfaces of the block.

When the Richardson number is 1.0, the flow configuration is affected by the forced convection effects due to the lid motion and the natural convection effects due to the buoyancy driven flow.

It can be seen in Fig. 5 that the arrangement of cold sources on the Top-Right is no longer the best arrangement, it has the largest Nusselt number on the hot wall when the cold sources are placed on the top and bottom walls. The streamline diagram of the six arrangements have begun to different. The position of the vortex in the upper right corner caused by top lid driven is significantly different. For the arrangement of Left-Bottom, few parts of vortex act on the right wall of the block, which causing sparese isotherms on the right side of the cavity. It incidates lower temperature gradient there, the heat transfer level on the right side of the cavity is weak. At this time, the values of Nusselt number in the right wall of the block is also minimum. For the arrangement of Top-Right, the area of vortex act on the right wall of the block becomes significantly large, it makes the heat transfer of the right wall strong, and the values of Nusselt number in the right wall of the block is maximum. The vortex caused by temperature difference locates in the left of the square cavity, all vortices act on the entire left wall of the block for six arrangements of the cold sources, and the intensity of the isotherm is more evenly in the left side of the block.

Top-Left

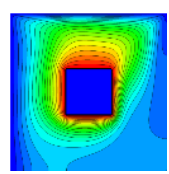

$\overline{N u}=7.76 \quad \Psi_{\min }=-0.28$

Left-Bottom

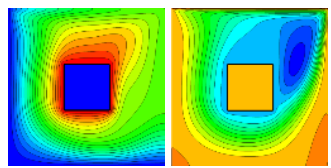

$\overline{N u}=4.37 \quad \Psi_{\min }=-0.27$

Top-Bottom

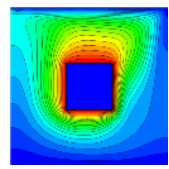

$\overline{N u}=7.85$

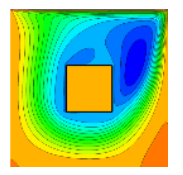

$\psi_{\min }=-0.24$
Left-Right

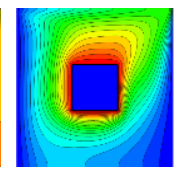

$\overline{N u}=6.76 \quad \Psi_{\min }=-0.28$

Top-Right

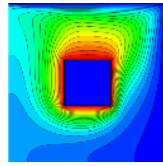

$\overline{N u}=8.06 \quad \psi_{\min }=-0.25$

Right-Bottom
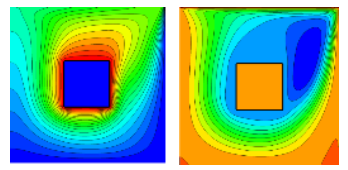

$\overline{N u}=6.66$

$\psi_{\min }=-0.24$

T: 0.050 .10 .150 .20 .250 .30 .350 .40 .450 .50 .550 .60 .650 .70 .750 .80 .850 .90 .95

Fig. 3. Streamlines and isotherms in the cavity with different arrangements of cold sources at $R i=0.1, G r=10^{5}$. 


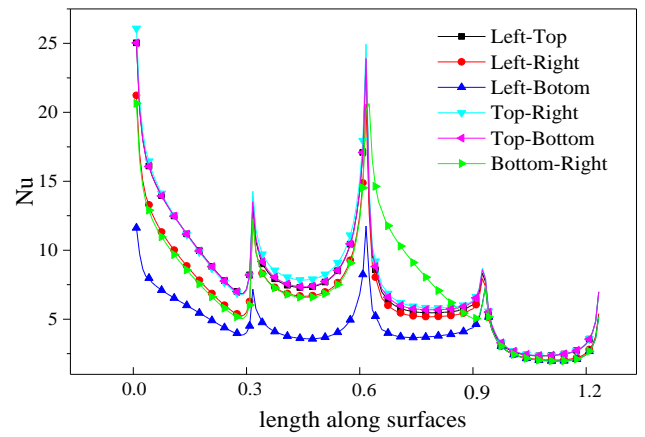

Fig. 4. Variation of the local Nusselt number along the edges of block at $R i=0.1, G r=10^{5}$.

Top-Left

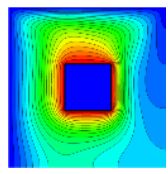

$\overline{N u}=6.42$

Left-Bottom
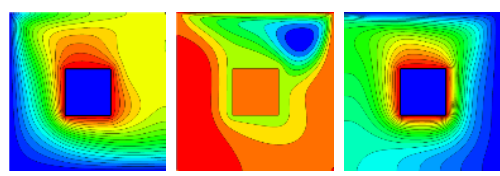

$\overline{N u}=4.27 \psi_{\min }=-0.45 \overline{N u}=6.37 \quad \psi_{\min }=-0.43$
Top-Bottom
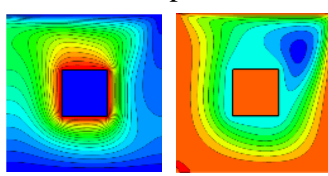

$\overline{N u}=6.58$
Left-Right

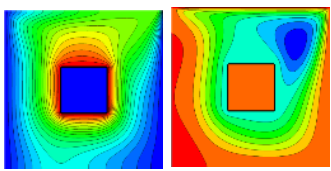

$\overline{N u}=5.84 \quad \psi_{\min }=-0.48$

Top-Right

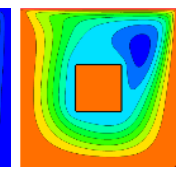

Right-Bottom

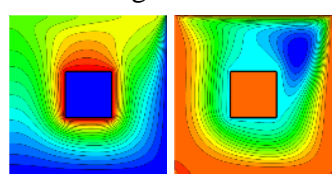

$\overline{N u}=5.64 \psi_{\min }=-0.38$

Fig. 5. Streamlines and isotherms in the cavity with different arrangements of cold sources at $R i=1.0, G r=10^{5}$.

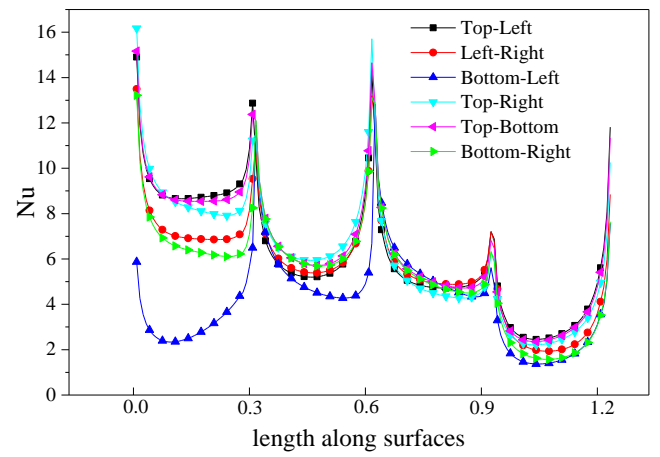

Fig.6. Variation of the local Nusselt number along the edges of block at $R i=1.0, G r=10^{5}$.
When the vaule of Richardson number is 10 , the flow pattern is dominaed by natural convection. Therefore, the arrangements of cold sources have a great influence on fluid flow and heat transfer in the cavity. As seen in Fig.7, the arrangements of the Top-Left, Left-Right, Left-Bottom form a flow pattern of two vortices, while other arrangements of cold sources form a vortex flow model. The arrangements of the Top-Left, Left-Right cold sources aid to form counterclockwise vortex caused by buoyancy force in the left side of the cavity. The combination of buoyancy and shear forces form a clockwise vortex on the right side of the cavity. The isotherms are more clustered on the left and right sides of the block for the arrangements of Top-Left, Left-Right. It has the largest Nusselt number on the hot walls when the cold sources are placed on the left and right walls. As seen in Fig.8, the Left-Right arrangement always display higher local Nusselt number on four surfaces of the block. For the arrangement of Left-Bottom, due to the limitation of arrangement of cold sources, the buoyancy flow can not be formed on the right side of the cavity, and the clockwise vortex is squeezed near the upper wall. This causes fluid flow and heat transfer weak on the right side of the cavity. The local Nusselt number on both right and top surfaces of the block are the lowest as shown in Fig.8 for the arrangement of Left-Bottom. This further illustrates that the heat transfer on the right side of the cavity is weak. Comparisons of streamlines and isotherms as shown in Fig.3, Fig.5 and Fig.7, streamlines and isotherms in the cavity change significantly with increasing $R i$. At lower $R i$ number, the flow pattern in the cavity is similar. With increasing $R i$, different numbers of vortex flow patterns are formed in the cavity for different arrangements of cold sources. This is due to the fact that buoyancy force caused by temperature difference domain the fluid flow. For the arrangement of Top-Left, the isotherm bends from upper right corner of the square cavity to upper left corner with $R i$ increasing. It can be explained that with $R i$ increases, the shear force caused by liddriven is no longer the main factor of fluid flow, the buoyancy force caused by temperature difference domain fluid flow. Counterclockwise vortex caused by buoyancy force in the left side of the cavity squeezes the clockwise vortex caused by shear force. The flow pattern in the cavity is changed from forced convection to natural convection, and the level of heat transfer is weakened, but the vortex intensity is enhanced in the cavity with the increasing of Richardson number. Several interesting features are observed in fluid flow and heat transfer. Heat transfer intensity in the cavity is relatively strong when one of a cold source is arranged on the upper wall of the square cavity. 
Top-Left

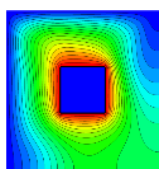

$\overline{N u}=5.18 \psi_{\max }=0.43$

$\psi_{\text {min }}=-0.46$

Left-Bottom

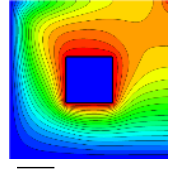

$\overline{N u}=4.29$

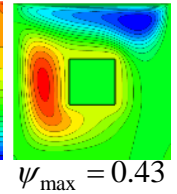

$\psi_{\text {min }}=-0.46$

Top-Bottom

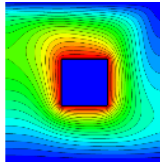

$\overline{N u}=5.36$

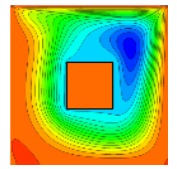

$\psi_{\min }=-0.88$

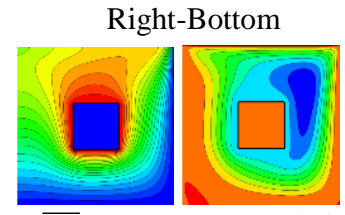

$\overline{N u}=4.88 \psi_{\min }=-0.45$

T: 0.050 .10 .150 .20 .250 .30 .350 .40 .450 .50 .550 .60 .650 .70 .750 .80 .850 .90 .95

Fig. 7. Streamlines and isotherms in the cavity with different arrangements of cold sources at $R i=10, G r=10^{5}$.

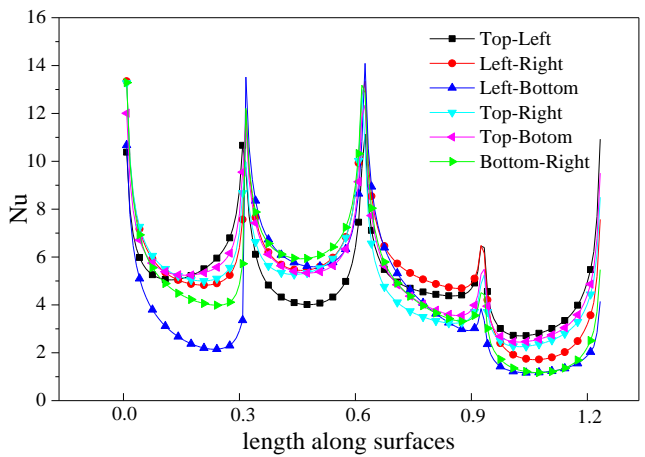

Fig. 8. Variation of the local Nusselt number

along the edges of block at $R i=10, G r=10^{5}$.

The variation of average Nusselt number on the surface of the block with different Richardson number under different arrangments of cold sources is shown in Fig.9. As for Top-Left, Top-Right and Top-Bottom arrangements, the average Nusselt number on the hot wall decreases linearly with the increasing of the Richardson number and they also show good heat transfer characteristics compared with other three arrangements. For the arrangement of Left-Bottom, the average Nuselt number of the hot wall surface is close to a horizontal line as the Richardson number changes. It also exhibits the worst heat transfer characteristics under the
Richardson number studied.

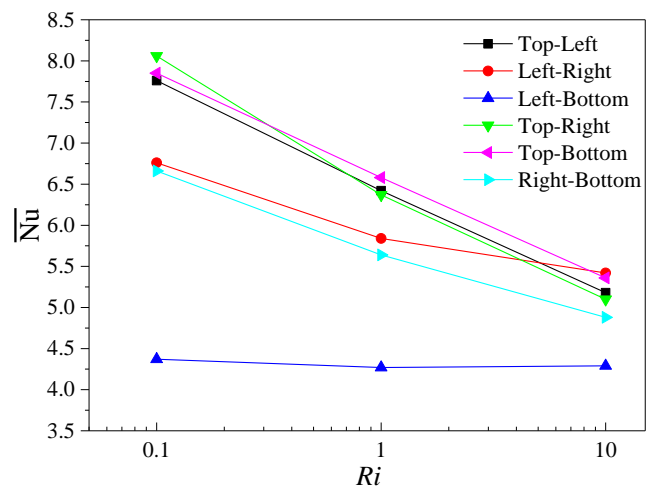

Fig. 9. Variation of average Nusselt number at the hot wall for different Richardson number.

\subsection{The Effect of Different Lid-Driven Pattern}

Four different cases of lid-driven on the surface of the cavity are discussed at different Richardson number. As seen in Fig. 10, only top wall moves rightwards and other walls remain stationary in case1. In case2, only bottom wall moves leftwards and other walls remain stationary. Both top and bottom walls move in the positive $\mathrm{X}$ direction for case3, while top and bottom walls move in the opposite direction in the case4. Temperature boundary conditions are same for in four cases. The top and bottom walls of the cavity are maintained at constant cold temperature while both vertical walls are thermally insulated.
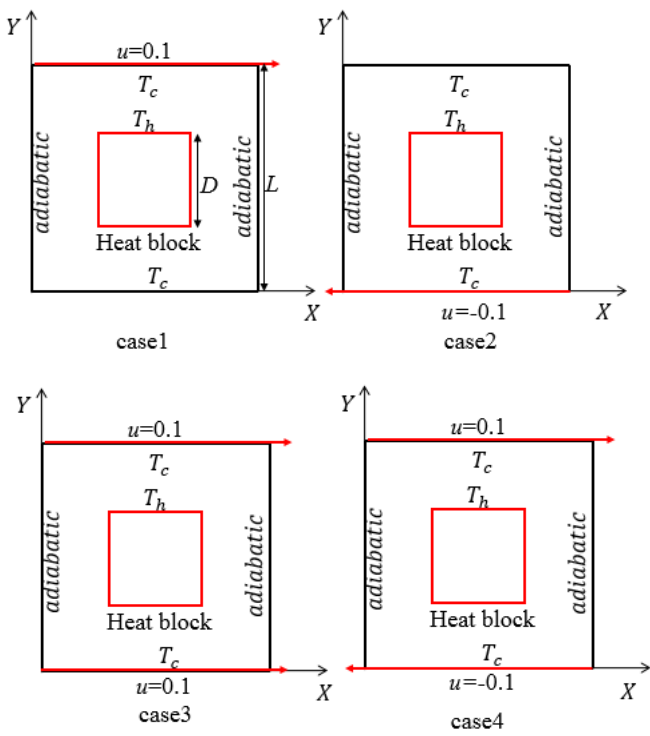

Fig. 10. Physical model for four cases of lid-driven.

The Richardson number is varied as $0.1,1.0,10$, corresponding to forced convection, mixed 
convection, natural convection dominated flow regimes. Figures 11, 14, 17 illustrate streamlines and isotherms in the cavity for $R i=0.1,1.0,10$, respectively. For a better understanding of flow characteristics in the cavity, the u-velocity in the vertical mid-plane and the v-velocity in the horizontal mid-plane are shown in Figs. 12, 15, 18 for $R i=0.1,1.0,10$, respectively. The variation of local Nusselt number along the hot wall of the heated block is shown in Figs. 13, 16, 19 with $R i=$ $0.1,1.0,10$. It starts from upper right corner of the block, and follow the right, bottom, left of the block, and then terminate at the top of the block. When the Richardson number is 0.1 , flow pattern of fluid in the cavity is forced convection, heat transfer and fluid flow in the cavity are dominated by shear force caused by lid-driven. Therefore, due to four different patterns of lid-driven, the streamlines and isotherms in the cavity vary greatly. It can be found in Fig.11 that a large clockwise vortex occours in the cavity due to the lid moving along X-direction for case1, and a tiny vortex occours in the right corner. For case2, the center of main vortex is located in the left of the block, this causes a large temperature gradient in the vicinity of left wall surface of the block. It can be seen in Fig.13, the local Nusselt number of the left wall surface is maximux. Tiny vortices can be seen in the streamlines of lower right and upper left corners of the case 1 and case 2 in the cavity, respectively. This is a common characteristic of the lid-driven cavity flow problem. Due to two lids moving in the same direction, two counter-rotating vortices appear in the cavity for case 3 . In the upper part of the cavity, lid-driven flow is rotated in clockwise direction. Buoyancy lead to an assisting effect on the movement of the top lid. However, for counterclockwise vortex, buoyancy lead to an opposite effect on the movement of the bottom lid. The clockwise vortex occupies the bulk of the cavity. Due to the flow separation of the vortex rotating clockwise direction, the vortex strength is weakened. The intensity of the vortex with counterclockwise rotating is higher than that of the vortex with clockwise rotating. The center of vortices are located on the right of the block, causing a large temprature gradient exist in the vicinity of the right wall surface of the block. Both right and top wall surfaces have biggest local Nusselt number, therefore the configuration of case 3 gives the maximum average Nussult values. As seen in Fig.11, a vortex with counterclockwise circulation disappears when two lids move in opposite directions for case4. Due to the effect of shear force outweighs that of buoyancy force, only a large clockwise vortex generates in the cavity. Fluid disturbance of the cavity become weakened,

which makes the heat transfer worse. Velocity profiles with $R i=0.1$ are presented in Fig.12, the minimum and maximum points can be observed in vertical velocity profiles.Furthermore, it is verified that a clockwise vortex is formed in the cavity for case4.

case 1

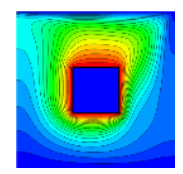

$\overline{N u}=7.85$

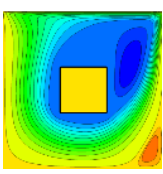

case 3

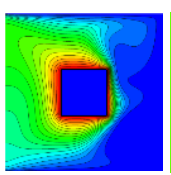

$\overline{N u}=10.14$

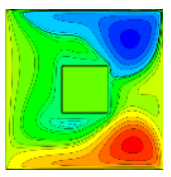

$\psi_{t, \min }=-0.26$ $\psi_{b, \max }=0.32$

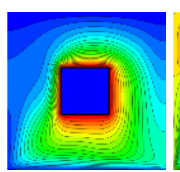

case2

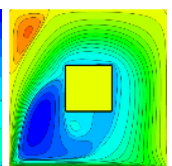

$\psi_{\min }=-0.28$

case 4

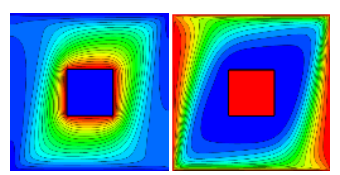

$\overline{N u}=6.39 \quad \psi_{\min }=-0.32$

Fig. 11. Streamlines and isotherms in the cavity for different lid-driven patterns at $R i=0.1$, $G r=10^{5}$.

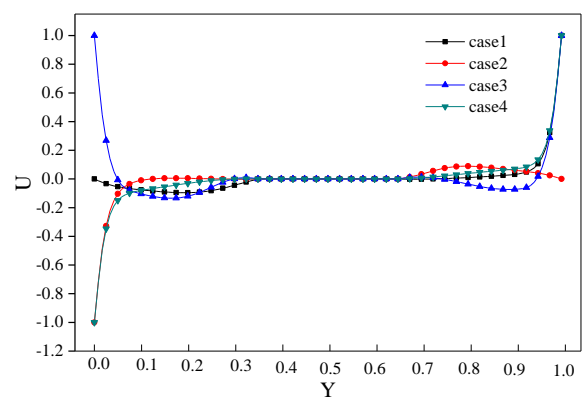

(a) U-velocity

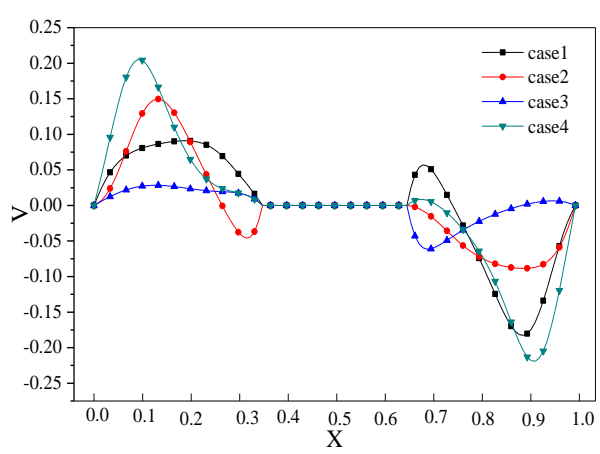

(b) V-velocity

Fig. 12. Velocity profiles along the vertical (a) and horizontal mid-plane (b) in the cavity with $R i=0.1, G r=10^{5}$.

When $R i$ is increased to 1.0 , the flow structure is affected by forced convection effects due to the lid 
motion and natural convection effects due to the buoyancy. Figure14 depict streamlines and isotherms in the cavity for four different models of lid-driven at $R i=1.0$. For case1, it forms a primary clockwise vortex that occupies entire cavity, which is smilar in Fig.11 for case1. Three vortices appear in the cavity for case2. Compared with Fig.11, the clockwise vortex that occupies most of the cavity becomes two vortices. It can be explained that with increases $R i$, the effect of buoyancy can no longer ignored compared to shear force. Due to increase buoyancy in the right side of the cavity, the clockwise vortex generated by the bottom liddriven is divided into two vortices. The bottom liddriven is insufficient to affect fluid flow in the upper left corner of the cavity. Therefore, The counterclockwise vortex in the upper left corner become bigger. As seen in Fig.15(a), the u-velocity distribution of case 2 shows a horizontal line closing to 0 in the upper portion of the cavity. This also indicates that bottom lid-driven have few influence on fluid flow in the upper portion of the cavity. And the vertical velocity distribution shows the peak of case 2 is lowest compared with other three cases. For case3, the vortex with clockwise rotating occupies bigger area of the cavity. The center of vortex begin to act on the upper right corner of the block, causing a large temperature difference in the vicinity of the right wall of the block. As seen in Fig.16, the local Nusselt number of both right and bottom walls of the block are higher, and the average Nusselt number is also biggest in case3. Under this condition, lid-driven of case 3 has the best heat transfer characteristics. For case4, isotherm doesn't bend in the direction of the liddriven as in Fig. 11, it bends at the four corners of the cavity. This is the result of the combination of buoyancy and shear force.

When $R i=10$, flow structure is dominated by natural convection. As shown in Fig.17, the shape and size of vortex changes. The shear force generated by the movement of top lid in positive $\mathrm{x}$-direction contributes to the formation of clockwise vortex for case1, case3, case4. And the intensity of clockwise vortices are the highest compared with $R i=0.1$ (see Fig.11) and $R i=1.0$ (see Fig.14). As for case2, natural convection contributes to upward flow of fliud in the vicinity of right wall of the block. When the clockwise vortex generated by bottom liddriven moves through the lower right of block, it merges with the vortex caused by buoyance force on the right side of the block. The center of clockwise vortex is seperated at the corner of block, causing sparse isotherms appeared in the right wall of the block. Since natural convection dominates fluid flow and heat transfer in the cavity, the clockwise vortex formed by the shear force is compressed near the bottom wall. For case4, isotherm bends toward to lid driven direction and cold fluid converges to the upper right and lower left corners of the cavity. The reverse movement of top and bottom walls contributes to the generation of vortex caused by natural convection. Figure 18 provides a more intuitive understanding of fluid flow in the cavity, patterns of double lid-driven significantly enchance fluid flow in the upper and lower portions of block compared with case 1 and case2. When $X>0.65$, vertical velocity increases from 0 to a positive peak value and then decreases to a negative peak value and finally increases to 0 for all cases. This indicates that clockwise vortices form in the upper portion of the cavity. As seen in Fig.19, the local Nusselt number of both top and left walls of the heated block are higher, and the average Nusselt number is also biggest in case4. Under this condition, case 4 have a better heat transfer rate.

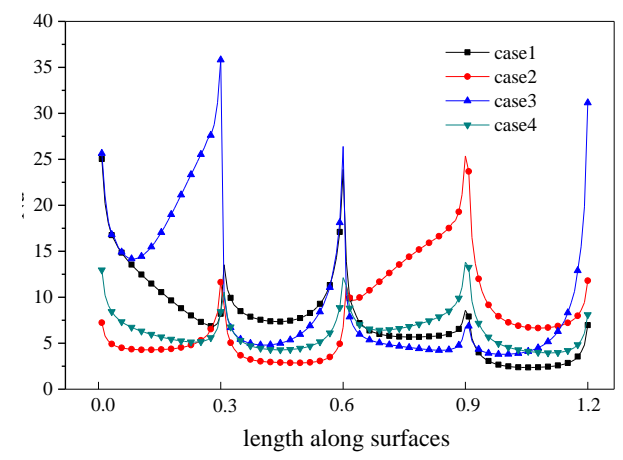

Fig. 13. Variation of the local Nusselt number along the edges of block at $R i=0.1, G r=10^{5}$.

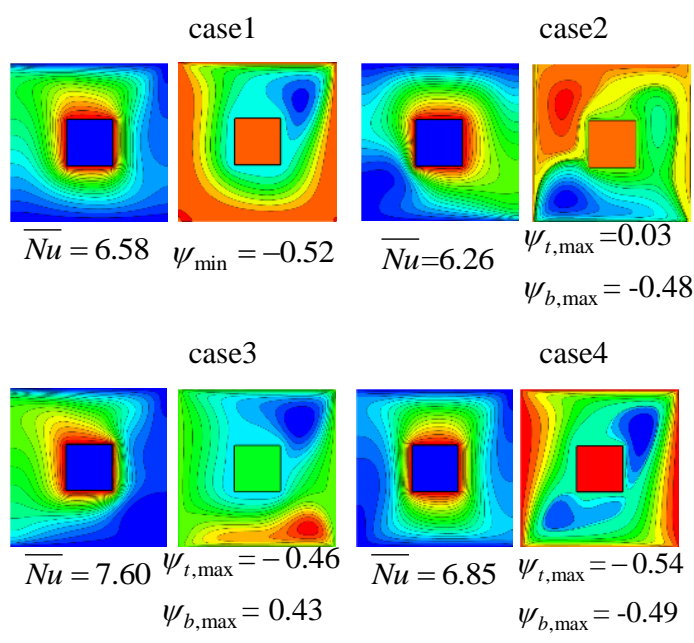

Fig. 14. Streamlines and isotherms in the cavity for different lid-driven patterns at $R i=1, G r=10^{5}$. 


\section{CONCLuSION}

In the present study, numerical simulations of

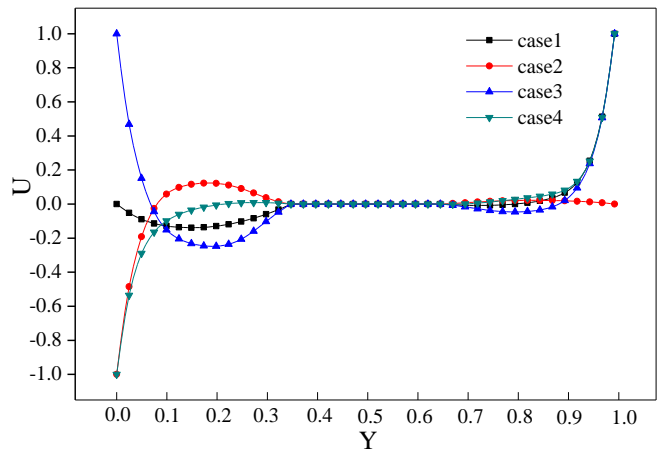

(a) U-velocity

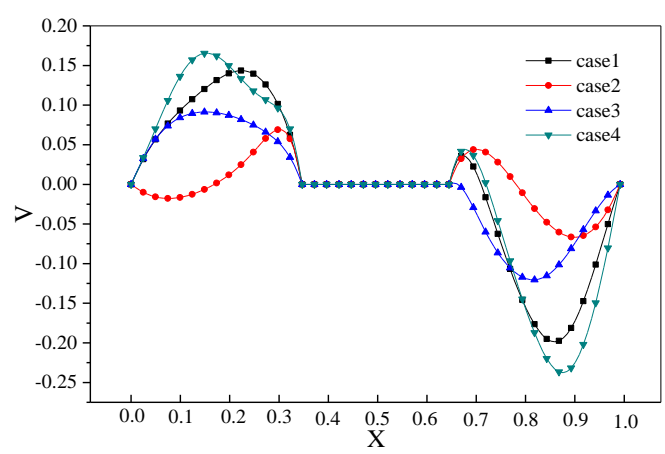

(b) V-velocity

Fig. 15. Velocity profiles along the vertical (a) and horizontal mid-plane (b) in the cavity with $R i=1.0, G r=10^{5}$.

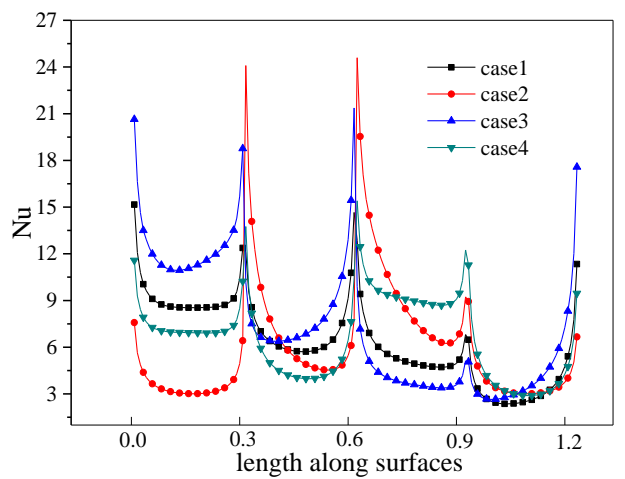

Fig. 16. Variation of the local Nusselt number along the edges of block at $R i=1.0, G r=10^{5}$.

laminar mixed convection in lid-driven porous cavity with an isothermally heated block are investigated by non-orthogonal multiple-relaxation time lattice Boltzmann method. The effects of different arrangements of the cold sources on the characteristics of fluid flow and heat transfer are investigated. In addition, the effects of different lid-

driven patterns on fluid flow and heat transfer are studied. From the numerical results, the following conclusions can be established:

case1

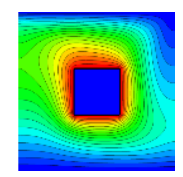

$\overline{N u}=5.36 \psi_{\min }=-0.88$

case 3

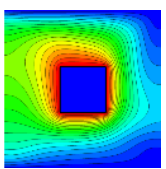

$\overline{N u}=5.54$

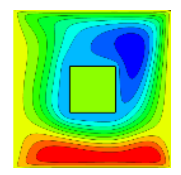

$\psi_{t, \min }=-0.8$

$\psi_{b, \max }=0.40$

Fig. 17. Streamlines and isotherms in the cavity for different lid-driven patterns at $\boldsymbol{R} i=10$, $G r=10^{5}$.

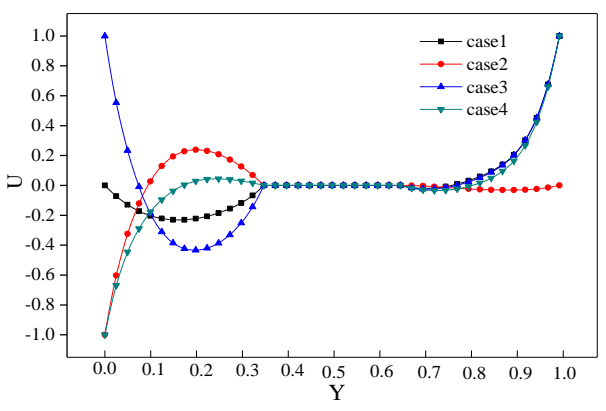

(a) U-velocity

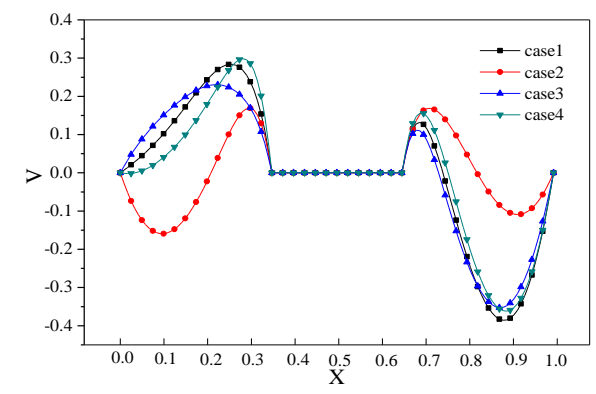

(b) V-velocity

Fig. 18. Velocity profiles along the horizontal (a) and vertical mid-plane (b) in the cavity with $R i=10, G r=10^{5}$.

As for the influence of cold source locations on fluid flow and heat transfer, it is found that the arrangements of the Top-Left, Left-Right, LeftBottom change the number of vortex in the cavity from one to two as the Richardson number increases, while only a vortex is formed in the cavity for other three arrangements. As for Top- 
Left、Top-Right and Top-Bottom arrangements, they always show high heat teansfer performance. For the arrangement of Left-Bottom, heat transfer characteristics do not change significantly with increasing Richardson number and it always display lowest average Nusselt value compared with other arrangements. When $R i<1$, the traditional left and right or up and down arrangements of the cold walls is no longer the optimal arrangement, the Top-Right arrangement displays high heat transfer characteristics. When the cold sources are arranged on the upper wall of the square cavity, it shows better heat transfer performance.

We also analyze the effects of the driving lid on fluid flow and heat transfer. The top and bottom walls maintain cold temperture codition. The results show that when $R i \leq 1$, the case of top and bottom walls move in opsitive $\mathrm{X}$ direction displays best heat transfer characteristic than other three cases. The case of Top and bottom wall move in the opposite direction has best heat transfer performance than other three cases when $R i>1$.

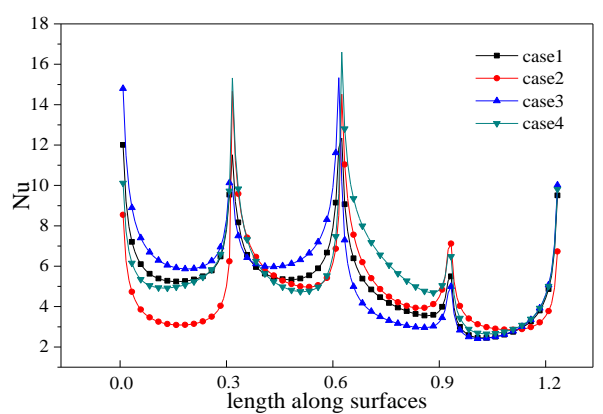

Fig. 19. Variation of the local Nusselt number along the edges of block at $R i=10, G r=10^{5}$.

\section{ACKNOWLEDGEMENTS}

This paper is supported by the National Natural Science Foundation of China (11562011) and the Natural Science Foundation of Jiangxi Province (20151BAB202002).

\section{REFERENCES}

Al-Amiri, A. M. (2000). Analysis of momentum and energy transfer in a lid-driven cavity filled with a porous isothermally heated block. International Journal of Heat and Mass Transfer 43(19),3513-3527.

Arun, S. and A. Satheesh (2015). Analysis of flow behaviour in a two sided lid driven cavity using lattice Boltzmann technique. Alexandria
Engineering 'Journal 54(4), S1110016815000976.

Billah, M. M., M. M. Rahman, U. M. Sharif, N. A. Rahim, R. Saidur and M. Hasanuzzaman (2011). Numerical analysis of fluid flow due to mixed convection in a lid-driven cavity having a heated circular hollow cylinder. Procedia Engineering 38(8), 1093-1103.

Chai, Z., Z. Guo and B. Shi (2007). Lattice Boltzmann Simulation of Mixed Convection in a Driven Cavity Packed with Porous Medium. International Conference on Computational Science. Springer-Verlag.

Cheng, T. S. and W. H. Liu (2010). Effect of temperature gradient orientation on the characteristics of mixed convection flow in a lid-driven square cavity. Computers and Fluids 39(6), 965-978.

Gangawane, K. M. and B. Manikandan (2017). Mixed convection characteristics in lid-driven cavity containing heated triangular block. Chinese Journal of Chemical Engineering (10), 43-56.

Guo, Z. and T. S. Zhao (2002). Lattice Boltzmann model for incompressible flows through porous media. Physical Review E 66(3 Pt 2B), 036304.

Islam, A. W., M. A. R. Sharif and E. S. Carlson (2012). Mixed convection in a lid driven square cavity with an isothermally heated square blockage inside. International Journal of Heat and Mass Transfer 55(19-20), 52445255 .

Iwatsu, R., J. M. Hyun and K. Kuwahara (1993). Mixed convection in a driven cavity with a stable vertical temperature gradient. International Journal of Heat and Mass Transfer, 36(6), 1601-1608.

Jonas, L., (2008). Choice of units in lattice Boltzmann simulations, Palabos LBM Wiki documentation project.

Kalteh, M., K. Javaherdeh and T. Azarbarzin (2014). Numerical solution of nanofluid mixed convection heat transfer in a lid-driven square cavity with a triangular heat source. Powder Technology, 253, 780-788.

Khali, S., R. Nebbali, D. Ameziani and K. Bouhadef (2013). Numerical investigation of non-newtonian fluids in annular ducts with finite aspect ratio using lattice Boltzmann method. Physical Review E, 87(5), 1-15. 
Koosukuntla, N. (2011). Towards development of a multiphase simulation model using lattice Boltzmann method (lbm), Master's thesis, University of Toledo, Toledo.

Li, P., J. Sun, Y. Zhang and W. Li (2018). Lattice Boltzmann simulation of natural convection in an inclined porous cavity with a hot square obstacle. Journal of Harbin Engineering University 39(6): 1073-1080.

Li, Q., Q. Kang, M. Francois, Y. He and K. Luo (2015). Lattice Boltzmann modeling of boiling heat transfer: the boiling curve and the effects of wettability. International Journal of Heat and Mass Transfer 85, 787-796.

Li, Q., Y. L. He, G. H. Tang and W. Q. Tao (2010). Improved axisymmetric lattice Boltzmann scheme. Physical Review E $81(5$ Pt 2), 056707.

Liu, Q. and Y. L. He (2015). Double multiplerelaxation-time lattice Boltzmann model for solid-liquid phase change with natural convection in porous media. Physica A: Statistical Mechanics and its Applications 438, 94-106.

Liu, Q., Y. L. He, D. Li and Q. Li (2016). Nonorthogonal multiple-relaxation-time lattice Boltzmann method for incompressible thermal flows. International Journal of Heat and Mass Transfer 102, 1334-1344.

Nebbali, R., S. Khali and K. Bouhadef (2017). Effect of a porous layer on newtonian and power-law fluids flows between rotating cylinders using lattice Boltzmann method. Journal of the Brazilian Society of
Mechanical Sciences and Engineering 39(10), 3881-3895.

Oztop, H. F., M. Oztop and Y. Varol (2009). Numerical simulation of magnetohydrodynamic buoyancy-induced flow in a non-isothermally heated square enclosure. Communications in Nonlinear Science and Numerical Simulation 14(3), 770778.

Premnath, K. N. and S. Banerjee (2009). Incorporating forcing terms in cascaded lattice Boltzmann approach by method of central moments. Physical Review E 80(3), 036702.

Qian, Y. H., D. D'Humières and P. Lallemand (1992). Lattice bgk models for navier-stokes equation. Europhysics Letters (EPL) 17(6), 479-484.

Sivakumar, V., S. Sivasankaran, P. Prakash and J. Lee (2010). Effect of heating location and size on mixed convection in lid-driven cavities. Computers and Mathematics with Applications 59(9), 3053-3065.

Vafai, K. (2006). Convective flow and heat transfer in variable-porosity media. Journal of Fluid Mechanics 147(147), 233-259.

Zhao, C. Y., L. N. Dai, G. H. Tang, Z. G. Qu and Z. Y. Li (2010). Numerical study of natural convection in porous media (metals) using lattice Boltzmann method ( $(\mathrm{bm})$. International Journal of Heat and Fluid Flow 31(5), 925934.

Zou, Q. and X. He (1997). On pressure and velocity boundary conditions for the lattice Boltzmann bgk model. Physics of Fluids 9(6), 1591-0. 